\title{
Reconstruction of Purine Metabolism in Bacillus subtilis to Obtain the Strain Producer of AICAR: A New Drug with a Wide Range of Therapeutic Applications
}

\author{
K.V. Lobanov, L. Errais Lopes, N.V. Korol'kova, B.V. Tyaglov, A.V. Glazunov, R.S. Shakulov, \\ A.S. Mironov* \\ State Research Institute for Genetics and Selection of Industrial Microorganisms \\ *E-mail: alexmir@genetika.ru \\ Received 24.02.2011
}

\begin{abstract}
AICAR is a natural compound, an analogue and precursor of adenosine. As activator of AMP-activated protein kinase (AMPK), AICAR has a broad therapeutic potential, since it normalizes the carbohydrate and lipid metabolism and inhibits the proliferation of tumor cells. The synthesis of AICAR in Bacillus subtilis cells is controlled by the enzymes of purine biosynthesis; their genes constituting purine operon (pur-operon). Reconstruction of purine metabolism in B. subtilis was performed to achieve overproduction of AICAR. For this purpose, the gene $\mathrm{purH}$, which encodes formyltransferase/IMP-cyclohydrolase, an enzyme that controls the conversion of AICAR to IMP, was removed from the B. subtilis genome, ensuring the accumulation of AICAR. An insertion inactivating the gene $p u r R$ that encodes the negative transcriptional regulator of the purine biosynthesis operon was introduced into the B.subtilis chromosome in order to boost the production of AICAR; the transcription attenuator located in the leader sequence of pur-operon was deleted. Furthermore, the expression integrative vector carrying a strong promoter of the $r p s F$ gene encoding the ribosomal protein S6 was designed. The heterologous Escherichia coli gene purF encoding the first enzyme of the biosynthesis of purines with impaired allosteric regulation, as well as the modified E.coli gene prs responsible for the synthesis of the precursor of purines - phosphoribosyl pyrophosphate (PRPP) - was cloned into this vector under the control of the rpsF gene promoter. The modified purF and prs genes were inserted into the chromosome of the B. subtilis strain. B. subtilis strain obtained by these genetic manipulations accumulates 11-13 $\mathrm{g} / \mathrm{L}$ of AICAR in the culture fluid.

KEYWORDS anticancer agent AICAR; purine metabolism; genome reconstruction; Bacillus subtilis strain - producer of AICAR

ABBREVIATIONS AICAR - 5-aminoimidazole-4-carboxamide ribofuranoside; AICAR-P - nucleotide AICAR phosphate; IMP - inosine monophosphate; AMP - adenosine monophosphate; AMPK - 5'-adenosine monophosphate-activated protein kinase; PRPP - phosphoribosyl pyrophosphate; GAR - 5'- phosphoribosyl glycineamide ribonucleotide; GIMP - guanosine monophosphate; PCR - polymerase chain reaction; CF - culture fluid
\end{abstract}

\section{INTRODUCTION}

Despite the fact that the structural organization of the genes encoding the enzymes of purine nucleotide biosynthesis is quite versatile, the biochemistry of the process is conservative for different organisms: the formation of the purine cycle occurs on the basis of a riboso-5-phosphate (all intermediates are nucleotides) using a monocarbon component (formiate and/or N10formyltetrahydrofolate) [1]. There is demand for monocarbon compounds at two stages of purine biosynthesis; therefore, the precursors - phosphoribosylglycineamide ribonucleoside (GAR) and 5-aminoimidazole-4carboxamide ribonucleoside (AICAR-P) can be accumulated if there is a deficiency in these compounds. Among them, AICAR-P occupies a specific place, since its formulation and subsequent cyclization crown the formation of the purine heterocycle that yields inosine monophosphate (IMP) (Fig. 1). The process of conversion of AICAR-P into IMP in prokaryotic cells is controlled by the gene purH, which encodes two domains with the activities of AICAR-P-formyltransferase and IMP cyclohydrolase [2, 3]. Further modifications of IMP yield AMP and GMP.

Despite the fact that the structure of purine heterocycle is incomplete, AICAR-P is a natural analogue of AMP, substituting it in certain enzymatic in vitro reactions. The possibility that AMP could be substituted in the reactions of activation of $\mathrm{AMP}$-activated proteinkinase (AMPK) in mammals has been given a significant degree of attention over the past decade. AMPK is 
the global regulator of the metabolic processes ensuring the energy status of the eukaryotic organism [4, 5]. For in vivo activation of AMPK, it is convenient to use a AICAR nucleoside, which can be rapidly phosphorylated in cells, yielding AICAR-P, an analogue of AMP. The emergence of AICAR-P imitates the accumulation of AMP and provokes the rearrangement of energy processes directed towards the overcoming of imaginary energetic stress. Due to their ability to activate AMPK, AICAR-based drugs have a broad therapeutic potential, since they normalize both the carbon [6] and lipid [7] metabolism. AICAR suppresses tumor cell growth by imitating the state of energetic stress [8]. The efficacy of AICAR in the prevention of type 2 diabetes mellitus has been demonstrated [9]. AICAR induces apoptosis; it is efficient upon chronic [10] and acute leukoses [11].

The present work was aimed at obtaining the strain producer of AICAR by directed reconstruction of purine metabolism in B. subtilis cells. The choice of B. subtilis was conditioned on the fact that genetic control and regulation of purine metabolism in these bacteria have been subjected to an appropriately thorough study. Furthermore, Bacillus strains have been in use for a significant length of time as producers of purine nucleosides and nucleotides, such as inosine and inosinic, as well as guanosinic, acids $[12,13]$.

Purine operon B. subtilis, purEKBCSQLFMNHD (hereafter referred to as pur-operon), encodes the enzymes of the synthesis of IMP, the most significant intermediate compound upon purine nucleotide biosynthesis (Fig. 1).

The group consisting of 12 linked genes that form the pur-operon is localized at the $55^{\circ}$ region on the chromosome of B. subtilis (Fig. 2) [14]. Expression of the pur-operon of $B$. subtilis undergoes a double- negative regulation, by the protein-repressor PurR [15] and the transcription attenuator located in the leader sequence of pur-operon [16]. It was shown that PRPP acts as a low-molecular-weight effector of the PurR protein [15], whereas guanine serves as a modulator enhancing transcription termination prior to the first structural gene of the operon [17]. Later, it was revealed that a 5'-non-translatable sequence of mRNA has a sensory function with respect to the metabolite - guanine effector, and that it acts as the so-called riboswitch [18, 19], providing early termination of operon transcription $[20,21]$.

Thus, maximum pur-operon gene expression had to be made possible at the first stage of obtainment of the strain producer of AICAR by eliminating the negative regulation of the pur-operon under the action of the protein repressor PurR and the transcription attenuator in the leader sequence of the operon. The gene

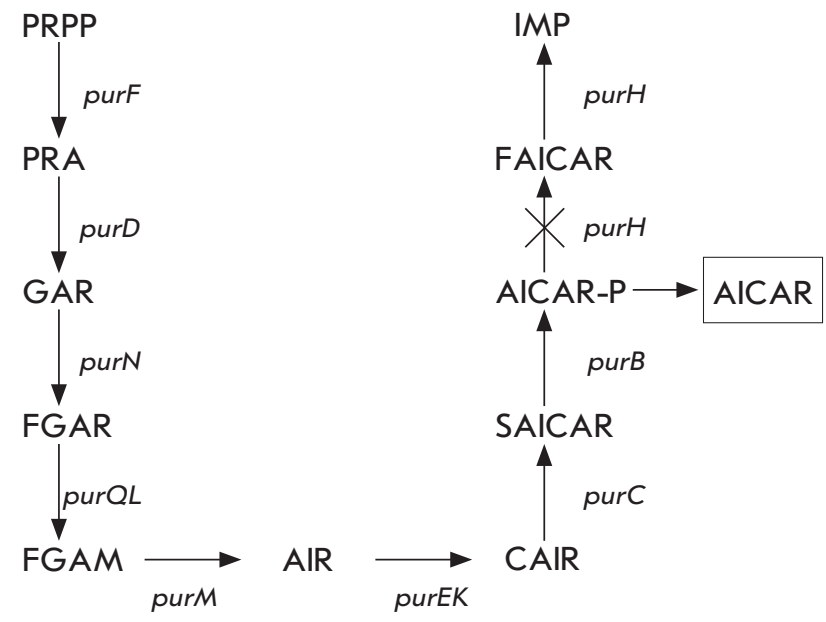

Fig. 1. De novo purine nucleotide biosynthesis in $B$. subtilis. Representative enzymatic steps of de novo purine biosynthesis are shown by the corresponding gene designations. Abbreviations: PRPP - 5'-phosphoribosyl-1-pyrophosphate, PRA-5'-phosphoribosylamine, GAR - 5'-phosphoribosylglycineamide, FGAR - 5'-phosphoribosyl-N-formylglycineamide, FGAM - 5'- phosphoribosyl-N-formylglycinamidine, AIR 5'-phosphoribosyl-5-aminoimidazole, CAIR - 5'-phosphoribosyl-4-carboxy-5-aminoimidazole, SAICAR - 5'-phosphoribosyl-4 (N- succinocarboxamide)-5-aminoimidazole, AICAR-P - 5' - phosphoribosyl - 4-carboxamide-5- aminoimidazole, FAICAR - 5-formamidoimidazole-4-carboxamide ribotide, IMP - inosine 5'-monophosphate, AICAR - 5-aminoimidazole-4-carboxamide 1- $\beta$-D-ribofuranoside.

purH encoding formyltransferase/IMP-cyclohydrolase, which participates in the synthesis of AICAR, was then deleted from the genome of the resulting strain. Inactivation of this enzyme is intended to ensure the intracellular accumulation of AICAR (Fig. 1). At the next stage, the pool of major precursors of de novo purine synthesis - PRPP - was to be increased. PRPP is synthesized from riboso-5-phosphate under the control of PRPP synthase encoded by the prs gene. This enzyme is susceptible to allosteric regulation with the participation of purine nucleotides. The structural and functional organization of PRPP synthase was studied more thoroughly in $E$. coli bacteria, in which a mutant variant of this enzyme, with eliminated allosteric regulation, was obtained [22]. Taking these data into account, site-directed mutagenesis of the prs gene of E. coli aimed at obtaining a mutant enzyme that would not be susceptible to retroinhibition by purine nucleotides was preformed with the purpose of its subsequent transfer into B. subtilis cells. .

An additional impediment in the effort to boost AIC$\mathrm{AR}$ production is the allostreric regulation of the first 


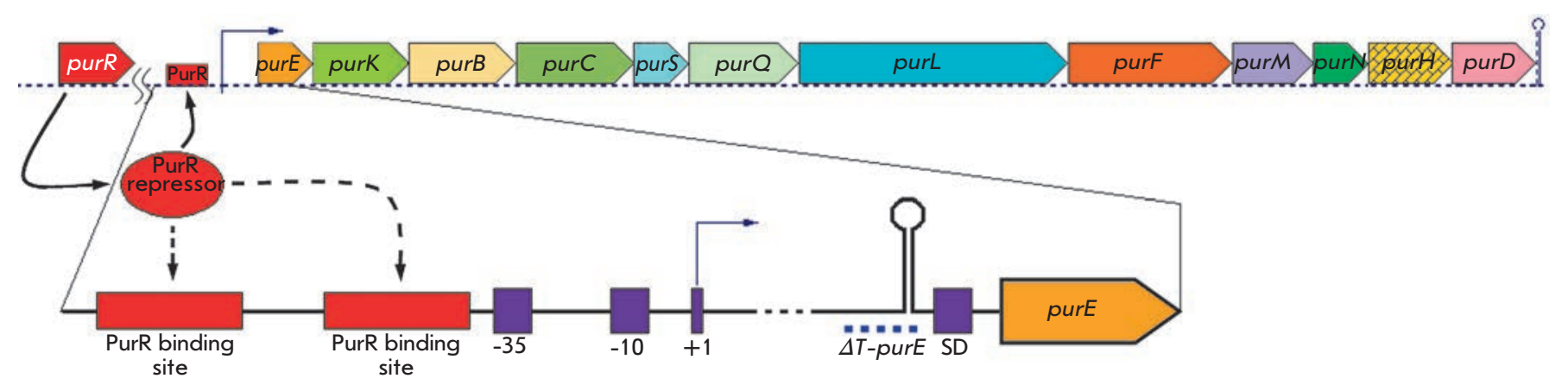

Fig. 2. Scheme of structural organization of the $B$. subtilis pur-operon and its regulation. Top: relative location of the 12 linked structural genes that constitute the pur-operon and unlinked purR gene, encoding a repressor of pur-operon. Bottom: the leader region of pur-operon, including binding sites for the repressor protein PurR, binding site for RNA polymerase $(-35 ;-10)$, transcription start $(+1)$, the terminator of transcription (hairpin structure) and the ribosome binding site (SD); the dotted line denotes deletion of the leader region of pur-operon $(\Delta T$-purE).

enzyme of purine biosynthesis - glutamine-PRPP aminotransferase encoded by the purF gene [23]. It is well known that glutamine-PRPP aminotransferase from $E$. coli, as opposed to the enzyme from B. subtilis, is not susceptible to inactivation in the steady-state stage of bacterial growth [24]. Moreover, the mutant variant of this enzyme, which is resistant to inhibition by purine nucleotides, has been described for $E$. coli [25]. Therefore, in our case the decision was made to use the glutamine-PRPP aminotransferase from $E$. coli modified by site-directed mutagenesis with the aim of subsequently transferring it into B. subtilis cells. An integrative expression vector based on plasmid pDG268 was constructed comprising a strong promoter of the rpsF gene, which encodes the ribosomal protein $\mathbf{S} 6$, in order to ensure the optimal expression of the modified prs and purF genes of E. coli in B. subtilis cells. The final stage of the process comprised the integration of the resulting vector, containing clones of the prs and purF genes under the control of the rpsF gene promoter, into the chromosome of the AICAR-producing strain B. subtilis.

\section{EXPERIMENTAL}

Bacterial strains and plasmids

The bacterial strains and plasmids used in this study are listed in Table 1. The B. subtilis strain AM732 was obtained via the transformation of the Mu8u5u6 strain of chromosomal DNA isolated from the B. subtilis strain 168 with the selection of $\mathrm{Pur}^{+}$transformants. Strain AM743 was obtained by the transformation of strain AM732 of chromosomal DNA isolated from B. subtilis strain LCC28 with the selection of transformants $\mathrm{Neo}^{\mathrm{R}}$ in a neomycine-containing medium.
Media and culturing conditions

A LB medium [31], or the standard-minimal Spizizen's medium, with the required additives [26] was used as the nutrient medium to culture bacteria. Aminoacids were added in an amount of $50 \mu \mathrm{g} / \mathrm{mL}$. Glucose $(0.4 \%)$ was used as a source of carbon. The following antibiotics were added into the medium when necessary: ampicillin (Amp) - $100 \mu \mathrm{g} / \mathrm{mL}$; chloramphenicol (Cm) - $10 \mu \mathrm{g} /$ $\mathrm{mL}$; erythromycin (Em) - $1 \mathrm{mg} / \mathrm{mL}$; and neomycin (Neo) $-5 \mu \mathrm{g} / \mathrm{mL}$. Methionine and leucine were added at a concentration of $50 \mu \mathrm{g} / \mathrm{mL}$. Hypoxantine, adenine, or guanine $(20 \mu \mathrm{g} / \mathrm{mL})$ was used as a purine source for purine auxotrophs. The DNA of $B$. subtilis was isolated according to the Saito-Miura procedure [32]; the transformation experiments were carried out in accordance with Anagnostopoulos and Spizizen's work [26].

\section{Manipulations with plasmid DNA}

The isolation of plasmid DNA, cloning, transformation into $E$. coli cells, and analysis of recombinant plasmids were performed using the standard methods [31].

\section{Enzyme preparations}

Restriction endonucleases, T4-DNA-ligase, and thermostable Taq DNA polymerase were purchased from Fermentas International Inc.

\section{Polymerase chain reaction}

The PCR was performed on a MyCycler thermal cycler (Bio-Rad Laboratories). The temperature mode was selected with due consideration of the length of the amplified fragment, as well as the length and composition of the primers used. The isolation and purification of the PCR products was carried out using a Silica Bead DNA Gel Extraction Kit (Fermentas International Inc.). 
Table 1. Bacteria and plasmids used in the present study

\begin{tabular}{|c|c|c|}
\hline Strain or plasmid & Description or genotype & Source or reference \\
\hline \multicolumn{3}{|l|}{ Bacillus subtilis } \\
\hline 168 & $\operatorname{trpC}$ & {$[26]$} \\
\hline Mu8u5u6 & leu met purF & {$[27]$} \\
\hline LCC28 & purR::neo & {$[28]$} \\
\hline AM747 & $\operatorname{trpC}$ purH::(pMutin2purH'-lacZ) $\Delta T$-purE & {$[21]$} \\
\hline AM732 & leu met & This study \\
\hline AM743 & leu met purR::neo & $-“-$ \\
\hline AM764 & leu met purR::neo $\Delta T$-purE & - “- \\
\hline AM778 & leu met purR::neo $\Delta T$-purE $\Delta$ purH & $-“-$ \\
\hline AM793 & leu met $\Delta$ purH & $-“-$ \\
\hline AM811 & leu met purR::neo $\Delta T-p u r E \Delta p u r H$ amyE::[P $\left.{ }_{r o s F}-p r s_{E}\right]$ & - “- \\
\hline AM813 & leu met purR::neo $\Delta T$-purE $\Delta$ purH amyE::[P $\left.P_{r n s F}-p u r F_{E}\right]$ & - “- \\
\hline AM815 & leu met purR::neo $\Delta T$-purE $\Delta T \Delta p u r H$ amyE::[P $\left[P_{r s s F}-p r s_{E^{-}}-p u r F_{E}\right]$ & - “- \\
\hline \multicolumn{3}{|c|}{ 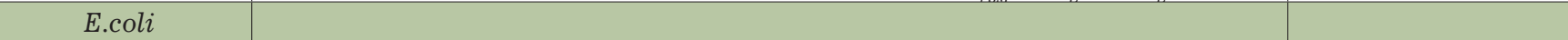 } \\
\hline TG1 & thi supE hsd $\Delta 5 \Delta\left(\right.$ lac-proAB)/F'tra $\Delta 36$ proAB $B^{(+)}$lac $I^{(q)} \operatorname{lacZ} \Delta M 15$ & VKPM \\
\hline MG1655 & prototroph & {$[29]$} \\
\hline \multicolumn{3}{|l|}{ Plasmids: } \\
\hline pDG268 & $\operatorname{Ap}^{\mathrm{r}}($ E.coli $) \mathrm{Cm}^{\mathrm{r}}$ (B.subtilis) & [18] \\
\hline pNZT1 & $\mathrm{Em}^{\mathrm{r}}$ & {$[30]$} \\
\hline pLE1 & as pDG268, but contains a promoter of $r p s F_{E}$ gene & This study \\
\hline pLE2 & as pLE1, but contains a prs $_{E}$ gene & $-“-$ \\
\hline pLE3 & as pLE1, but contains a $p u r F_{E}$ gene & - “- \\
\hline pLE4 & as pLE1, but contains a prs $_{E}$ и purF $F_{E}$ genes & $-“-$ \\
\hline
\end{tabular}

$A p^{r}$ - ampicillin resistance, $\mathrm{Em}^{r}$ erythromycin resistance, $\mathrm{Cm}^{r}$ - chloramphenicol resistance.

VKPM Russian National Collection of Industrial Microorganisms

Site-directed mutagenesis

Site-directed mutagenesis was performed using specific oligonucleotide primers. The composition and characteristics of the primers are listed in Table 2 . The presence of corresponding mutations was verified by sequencing according to Sanger [33].

\section{Fermentation conditions}

The ability of the strains obtained during the study to accumulate AICAR in culture liquid (CL) was assessed. The strain inoculum was cultivated at $37^{\circ} \mathrm{C}$ for $18 \mathrm{~h}$ on a LB broth. Then, $0.5 \mathrm{~mL}$ of the culture was added to each $20 \times 200 \mathrm{~mm}$ vial with $4.5 \mathrm{~mL}$ of the fermentation medium and cultured at $37^{\circ} \mathrm{C}$ for $72 \mathrm{~h}$ on a rotary shaker. The fermentation medium had the following composition (\%): soy flour - 3; nutrient yeast - 1; corn extract $-5 ;\left(\mathrm{NH}_{4}\right)_{2} \mathrm{HPO}_{4}-0.6$; carbamide -0.4 ; and sugar - 15, pH 7.0.

Determination of AICAR concentration in the culture liquid

The CL obtained during fermentation was centrifuged to remove the cells; the AICAR concentration was then determined in the supernatant on Sorbfil plates (OOO Lenchrom, Saint Petersburg, Russia) by quantitative thin-layer chromatography. The composition of the eluting system designed was as follows: chloroformmethanol-water-25\% aqueous solution of ammonia at a volume ratio of $5: 3: 1$. Quantitative HPLC on a chromatograph (ALLIANCE, Separations Module Waters 2695, Photodiode Array detector Waters 2996) was employed as an alternative method.

\section{RESULTS AND DISCUSSION}

Enhancement of the expression of the B. subtilis puroperon

In work [21] devoted to the study of B. subtilis puroperon expression, it was demonstrated that an almost 20-fold enhancement of the expression of the lac $Z$ reporter gene integrated into the $p u r H$ gene, as compared with the expression in the wild-type strain when the purR gene that encodes the repressor protein of the operon is damaged, is achieved. After deletion of 94 n.p. of the wild-type strain, which captures the Rho-independent transcription attenuator ( $\Delta T$-purE) 
Table 2. Primers used in this study

\begin{tabular}{|c|c|c|c|c|}
\hline \multirow{2}{*}{ Name } & \multirow{2}{*}{ Gene } & \multirow{2}{*}{ Sequence* } & \multicolumn{2}{|c|}{ Coordinates ** } \\
\hline & & & 5 & 3 \\
\hline N1 & purN $N_{B}$ & cccccgcgggcggaacgattccacat (SacII) & +135 & +154 \\
\hline $\mathrm{N} 2$ & $\operatorname{purN}_{B}$ & cgcctgcagttcttttacgaaaggaacga (PstI) & +652 & +630 \\
\hline D1 & purD $D_{B}$ & cgcctgcagcttcaaacattaaggggatgaaaa(PstI) & -28 & -5 \\
\hline D2 & purD $D_{B}$ & cgcggtacctttttcctgcacatatgcc (KpnI) & +410 & +389 \\
\hline $\mathrm{F} 1$ & purF $F_{E}$ & cgcatcgataggaggtgcaaacagatgtgcggtattgtcggtatc (ClaI) & +1 & +22 \\
\hline $\mathrm{F} 2$ & purF $F_{E}$ & cgcgctcagcgaaggcatcatcct (EspI) & +1530 & +1511 \\
\hline F3 & purF $F_{E}$ & gggcttcgttCaaaaccgctat & +968 & +991 \\
\hline F4 & purF $F_{E}$ & atagcggttttGaacgaagccc & +991 & +968 \\
\hline F5 & purF $F_{E}$ & ggtattgatatgTGgagcgccacgg & +1216 & +1242 \\
\hline F6 & purF $F_{E}$ & ccgtggcgctcCAcatatcaatacc & +1242 & +1216 \\
\hline $\mathrm{P} 1$ & prs $_{E}$ & cgcggatccaaggaggttcttctcAtgcctgatatga (BamHI) & -21 & +3 \\
\hline $\mathrm{P} 2$ & $\operatorname{prs}_{E}$ & cccatcgatgccgggttcgattagtgttcga (ClaI) & +949 & +928 \\
\hline P3 & prs $_{E}$ & ctgacagtgg $\mathbf{C t c t g c a c g c t g}$ & +366 & +377 \\
\hline $\mathrm{P} 4$ & prs $_{E}$ & agcgtgcagaGccactgtcagc & +377 & +366 \\
\hline $\mathrm{R} 1$ & $r p s F_{B}$ & cgcgaattcttgcgggcggcggtat (EcoRI) & -223 & -205 \\
\hline $\mathrm{R} 2$ & $r p s F_{B}$ & cgcggatccataatgggcaaggagcaat (BamHI) & -31 & -51 \\
\hline
\end{tabular}

${ }^{*}$ The sequence of primers is given in the orientation 5'-3'. Uppercase bold letters indicate the nucleotide substitutions introduced in primers for site-directed mutagenesis. Recognition sites are underlined. Restriction enzymes are shown in parentheses.

${ }^{* *}$ The coordinates of the 5'-and $3^{\prime}$-ends of the primers are relative to the start of translation of the corresponding genes. $B$. subtilis genes are marked with the symbol ${ }_{(\mathrm{B})}$; and $E$. coli - with the symbol ${ }_{(\mathrm{E})}$.

located in the leader region of the pur-operon (Fig. 2), the expression of the lac $Z$ reporter gene increased approximately by a factor of 10 . However, when combining both mutations, a pronounced synergic effect was observed; lac $Z$ gene expression increased by a factor of more than 200. Taking these data into account, the experiments on the inactivation of the purR gene and deletion of the transcription attenuator ( $\Delta T-p u r E)$ were performed at the first stage of construction of the AICAR-producing strain.

The AM732-Pur ${ }^{+}$strain, a derivative of the earlier characterized Mu8u5u6 strain [21] (Table 1), was used as starting material. In order to transfer the purR::neo insertion, which completely inactivates the synthesis of the PurR repressor protein, into the genome of the AM732 strain, this strain was transformed by chromosomal DNA isolated from the LCC28 (purR::neo) strain, with the selection of recombinants that were resistant to neomycin $\left(\mathrm{Neo}^{\mathrm{R}}\right)$. As a result, the AM743 purR::neo strain was selected for use in the subsequent work (Table 1). The deletion $\Delta T$-purE in the genome of the AM743 purR::neo strain was obtained through the following scheme. First, the long-stretched deletion $\Delta L-E$ was obtained, completely overlapping the leader region of the pur-operon and partially overlapping the first structural gene purE, which resulted in the emer- gence of auxotrophicity with respect to purines. How to achieve this deletion was thoroughly described in [21]. The deletion of the transcription attenuator $\Delta T$ purE was transferred into the AM743 purR::neo $\Delta L-E$ strain by transforming DNA that was isolated from the AM747 strain (Table 1); Pur ${ }^{+}$transformants were selected on the purine-free Spizizen's minimal medium. As a result, the AM764 strain was obtained. It contained the purR::neo insertion and deletion of the transcription attenuator $\Delta T$-purE in the genome.

Obtaining the deletion of the purH gene in the B. subtilis chromosome

As follows from the schematic representation of purine biosynthesis (Fig. 1), for intracellular accumulation of AICAR, it is necessary to inactivate formyltransferase/ IMP-cyclohydrolase encoded by the purH gene. The PurH gene deletion in the B. subtilis chromosome was achieved using the method described in [30] based on using a specially constructed pNZT1plasmid, a derivative of the integrative vector pKS1 [34]. The pNZT1 plasmid contains the erythromycine $\left(\mathrm{Em}^{\mathrm{R}}\right)$ resistance marker and a polylinker with multiple restriction sites for cloning the target fragments of chromosomal DNA. Of paramount importance is the fact that its replication is temperature-sensitive: at $30^{\circ} \mathrm{C}$, the plasmid exists in 
Fig. 3. A schematic representation of isolation of the purH gene deletion using the method described in [30].

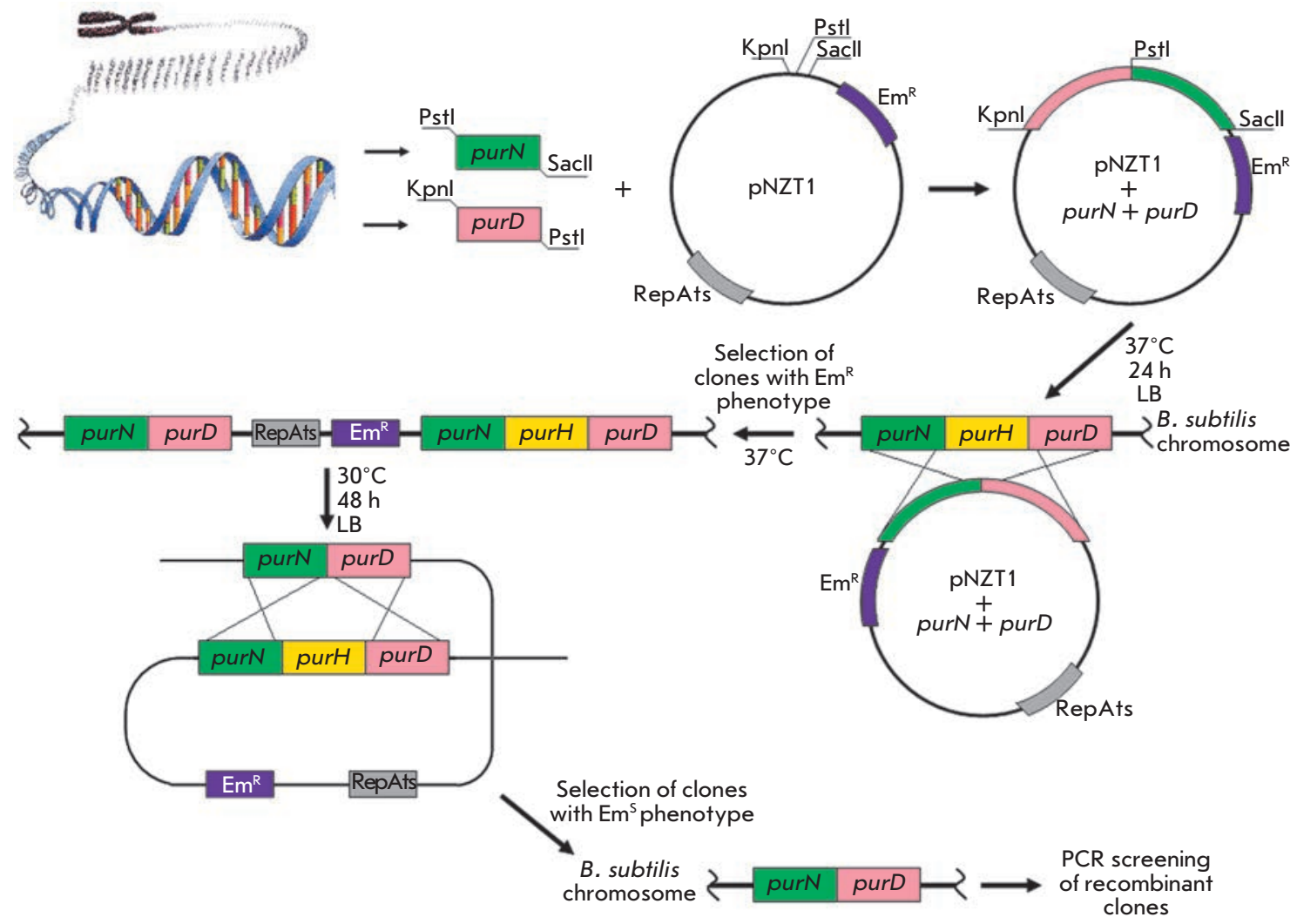

an autonomous state. However, at $37^{\circ} \mathrm{C}$ the replication is blocked. As a result, if the plasmid has a chromosome fragment cloned into its structure, it is capable of integration into the chromosome by homologous recombination (Fig. 3). Since plasmid integration into the chromosome results from the single recombination act in the homology region between the cloned fragment and the chromosome, the integration site of the plasmid into the chromosome turns out to be flanked by homologous duplicated sequences. Culturing of these bacteria at $30^{\circ} \mathrm{C}$ (permissive, for pNZT1 plasmid replication) may result in its removal from the chromosome with capture of a copy of the flanked chromosome sequences, which allows for the replacement of the wild allele of any chromosome gene by the mutant gene cloned in the pNZT1 plasmid (Fig. 3).

This feature of the pNZT1 plasmid was used for the deletion of the purH gene. With this aim in mind, the PCR amplification of the chromosome fragments that flanked the purH gene was performed. A distal region (517 np) of the purN gene adjacent to the 5'-terminus of the $p u r \mathrm{H}$ gene was amplified using N1 and N2 primers, followed by cloning into pNZT1 plasmid at restrictases SacII and PstI digestion sites. The proximal region (442 $\mathrm{np}$ ) of the purD gene adjacent to the 3'-terminus of the pur $\mathrm{H}$ gene was amplified at the next stage using D1 and D2 primers (Table 2), followed by cloning at restrictases PstI and KpnI digestion sites into the pNZT1 plasmid that was obtained at the previous stage and contained the insertion of the purN gene fragment. The E. coli strain TG1 was transformed by this plasmid. $\mathrm{Em}^{\mathrm{R}}$ transformants were sampled from a medium with erythromycin $(300 \mu \mathrm{g} / \mathrm{mL})$ at $30^{\circ} \mathrm{C}$. The presence of the pNZT1 plasmid with cloned purN and purD gene fragments was tested in the resulting clones using PCR. The AM764 purR::neo $\Delta T$-purE strain was transformed by pNZT1-purN-purD plasmids isolated from the inspected clones. The selection of the $\mathrm{Em}^{\mathrm{R}}$ transformants was carried out on a medium containing erythromycin $(3 \mu \mathrm{g} / \mathrm{mL})$. Several $\mathrm{Em}^{\mathrm{R}}$ clones were cultured at $37^{\circ} \mathrm{C}$ for a night, seeded into plates containing the LB medium with erythromycin $(3 \mu \mathrm{g} / \mathrm{mL})$ and incubated for $24 \mathrm{~h}$ at $37^{\circ} \mathrm{C}$. $\mathrm{Em}^{\mathrm{R}}$ recombinants were formed by integration of the pNZT1-purN-purD plasmid into the corresponding chromosome locus, which was attested by PCR amplification of the fragment consisting of 2413 bp using N1 and D2 primers. Several Em ${ }^{\mathrm{R}}$ clones were seeded into an antibiotic-free liquid LB medium and incubated on a shaker at $30^{\circ} \mathrm{C}$ for $48 \mathrm{~h}$. This was followed by seeding onto plates with an antibiotic-free LB medium and incubation for another $24 \mathrm{~h}$ at $30^{\circ} \mathrm{C}$. The excision of the integrated pNZT1 plasmid from the 
resulting clones was tested. This was detected by the emergence of erythromycin-sensitive clones. As noted above, plasmid excision can be accompanied by either the retention of the wild-type allele of the purH gene in the chromosome, or by substitution of this allele for the $\Delta$ purH deletion (Fig. 3). The integration (transfer) of the $\Delta$ purH deletion into the chromosome of the AM764 purR::neo $\Delta T$-purE strain was attested by the production of a PCR fragment (with a size of $2413 \mathrm{np}$ ) with the participation of $\mathrm{N} 1$ and $\mathrm{D} 2$ primers. One of the variants of the AM764 purR::neo $\Delta T$-purE strain comprising the $\Delta$ purH deletion and called AM778 was capable of accumulating up to $4-5 \mathrm{~g} / \mathrm{L}$ of AICAR in CL and grew on the minimal medium only upon addition of hypoxantine, adenine, or guanine, suchwise the presence of the defect purH gene in its genome was confirmed. Simultaneously, the control strain AM793 was constructed: it contained $\triangle p u r H$, but it carried no purR::neo and $\Delta T$-purE mutations, which provide derepression of the enzymes that involved in purine biosynthesis.

It was reasonable to increase the intracellular content of PRPP, the key precursor of purine biosynthesis; in order to enhance the productivity of the strain obtained (Fig. 1). It is well known that PRPP synthases that are responsible for PRPP synthesis in the cell of both B. subtilis [35] and E. coli [36] are susceptible to allosteric inhibition by purine nucleotides, including phosphorylated derivatives of AICAR. As previously mentioned in the introduction section, the mutant variant of PRPP synthase of $E$. coli, which is not susceptible to allosteric regulation, has been described [22]. Therefore, the goal was to obtain an analogous mutant enzyme of $E$. coli, to clone it, and to optimize the expression of this enzyme in strain AM778 cells. However, prior to this, the integrative expression vector had to be constructed, which could provide a high level of expression of heterologous E. coli genes in B. subtilis cells.

Construction of the integrative expression vector based on pDG268 plasmid

The pDG268 plasmid was selected as starting material in order to obtain the integrative expression vector. This plasmid can be replicated in $E$. coli (but not B. subtilis) cells. However, when introduced into B. subtilis cells, it may integrate into the chromosome locus amyE of B. subtilis. The pDG268 plasmid comprises a cartridge that includes the polylinker, lac $Z$ reporter gene without the intrinsic promoter, and the chloramphenicol $\left(\mathrm{Cm}^{\mathrm{R}}\right)$ resistance gene (Fig. 4). The cartridge is flanked by fragments of the amyE gene, which permits integration of the vector with the cloned fragment into the amyE locus on the B. subtilis chromosome.

The promoter of the rpsF gene encoding the ribosomal protein S6 in bacilla was selected as a promoter

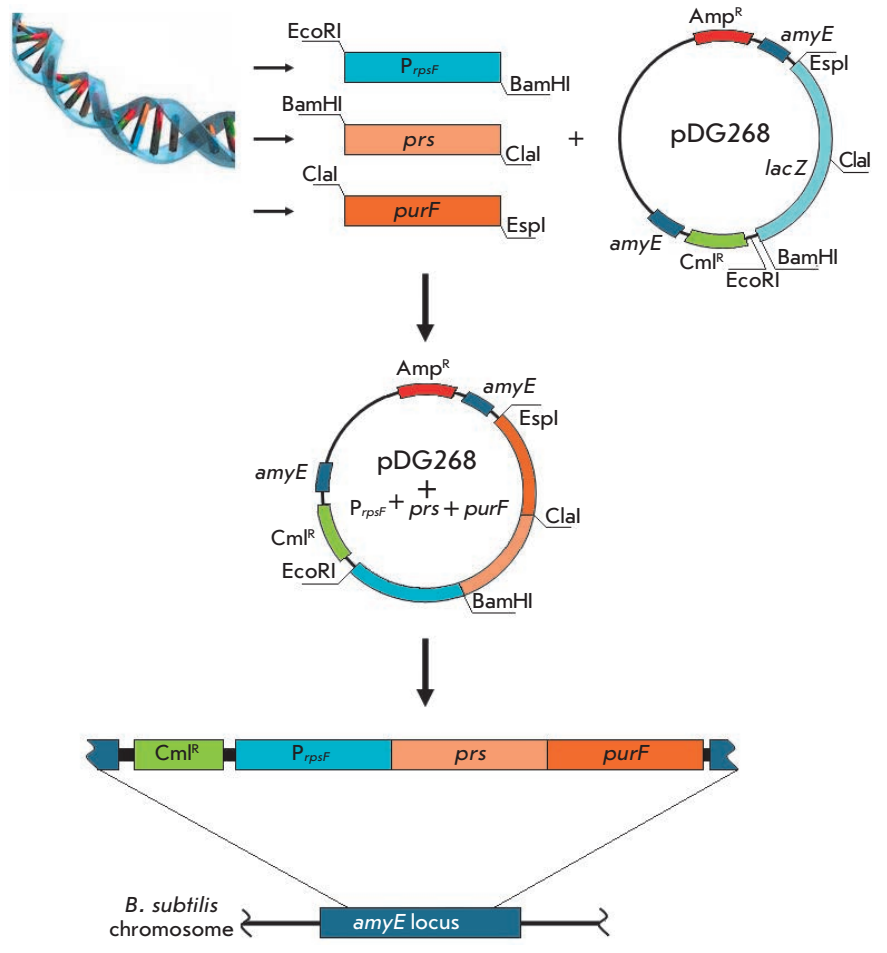

Fig. 4. A schematic representation of cloning procedure of $E$. coli prs and purF genes under the control of the B. subtilis rpsF gene promoter in the plasmid pDG268, and their integration into the $B$. subtilis chromosome.

that is capable of providing a high level of expression of the cloned E. coli genes in B. subtilis cells. The promoters of ribosomal protein genes have been known to belong to the strongest promoters in B. subtilis; their expression is coordinated with the bacterial growth rate and attains a maximum level at the logarithmic growth stage $[37,38]$. The nucleotide sequence of the rpsF gene promoter is shown in Fig. 5. As follows from Fig. 5, the $\mathrm{P}_{r p s F}$ promoter contains the canonic sequence $-70 \ldots-50 \mathrm{np}$, the so-called UP element, which provides a more efficient transcription initiation [39].

With the purpose of cloning the $\mathrm{P}_{r p s F}$ promoter into the pDG268 plasmid, the DNA fragment containing the promoter region of the $r p s F$ gene was amplified by PCR from the B. subtilis 168 chromosome using R1 and $\mathrm{R} 2$ primers (Table 2). The resulting PCR fragment was digested with the restriction endonucleases EcoRl and BamHI and cloned into the pDG268 plasmid digested with the same restrictases (Fig. 4). The E. coli TG1 strain was transformed by a ligase mixture. The transformants carrying the insertion of the desired fragment were sampled from an indicator medium containing ampicillin at a concentration of $120 \mu \mathrm{g} / \mathrm{mL}$ and X-gal. Transformant colonies were bright blue, since it was revealed that the lac $Z$ reporter gene was controlled by 
the cloned $\mathrm{P}_{r p s F}$ promoter. The presence of the insertion was confirmed by PCR using R1 and R2 primers. The resulting plasmid was then integrated into the chromosome amyE locus of $B$. subtilis by selecting the chloramphenicol $\left(\mathrm{Cm}^{\mathrm{R}}\right)$-resistent transformants. The schematic representation of the integration of the pDG268 vector with the cloned $B$. subtilis rps $F$ gene promoter is provided in Fig. 4. The determination of the activity of $\beta$-galactosidase in these transformants revealed that the lac $Z$ reporter gene expression under control of the $\mathrm{P}_{r p s F}$ promoter was higher than the expression of this gene when controlled by common promoters, such as the natural pur-operon promoter (no data presented), by an order of magnitude. The vector constructed was named pLE1.

Site-directed mutagenesis of the prs E. coli ( $\left.\operatorname{prs}_{E}\right)$ gene

According to the published data, specific mutation in the prs $_{E}$ gene, resulting in the Asp128 $\rightarrow$ Ala replacement in PRPP synthase, leads to the removal of retroinhibition of the enzyme by purine nucleotides [22]. In order to achieve the analogous mutation, synthesis of the oligonucleotide primers P3 and P4 (Table 2) was performed; the primers contain nucleotide replacements (are denoted by uppercase letters) resulting in the formation of a mutant protein with the Asp128 $\rightarrow$ Ala replacement. At the first stage, PCR fragments were amplified with the participation of two primer pairs: P4-P1 (it flanks the 5'-terminus of the prs gene and contains the recognition site for restrictase ClaI). Then, the obtained fragments were joined, and the full-size prs $_{E}$ gene was amplified using the P1 and P2 primers (Table 2). It should be emphasized that the nucleotide sequence of the ribosome recognition site (SD) optimized for expression in bacilli is included into the 5'-region of the $\mathrm{P} 1$ primer. At the next stage, the P1-P2 PCR fragment containing the mutant prs $_{E}$ gene was cloned into the earlier obtained pLE1 vector using BamHI and ClaI restrictases. As a result, pLE2 plasmid containing the mutant $\operatorname{prs}_{E}$ gene controlled by the $\mathrm{P}_{r p s F}$ promoter was obtained. At the final stage, pLE2 plasmid was integrated into the amyE B. subtilis chromosome locus according to the earlier described scheme (Fig. 4). The strain obtained by integration of the pLE2 plasmid was named AM811 (Table 1).

Site-directed mutagenesis of the purF E. coli $\left(p_{u r F}\right)$ gene

The first enzyme of purine biosynthesis, glutaminePRPP aminotransferase ( $p u r F$ gene), plays the primary role in ensuring the normal functioning of the purine biosynthetic path in E. coli and B. subtilis. In addition, it is susceptible to allosteric regulation with the participa- tion of nucleotides, which is even more sophisticated in comparison with the PRPP synthase [40]. Therefore, it could be expected that the use of the mutant glutaminPRPP aminotransferase from $E$. coli that is analogous to that described in [25] will lead to AICAR production. According to [25], Lys326 $\rightarrow$ Gln and Pro410 $\rightarrow$ Trp replacements in the protein modify the GMP (A-site) and AMP binding site (C-site), respectively. Combination of these mutations leads to the enzyme being resistant to almost any purine nucleotide. Site-directed mutagenesis of the $\operatorname{purF}_{E}$ gene was performed according to the scheme described in the previous section. The F3 and F4 oligonucleotide primers were synthesized to obtain the Lys326 $\rightarrow$ Gln replacement; and F4 and F5 primers, to obtain the Lys $326 \rightarrow$ Gln replacement (in Table 2, the corresponding nucleotide replacements are highlighted by uppercase letters and set off in bold). After the PCR amplification of these primers with the flanking primers F1 and F2, followed by the joining of the PCR fragments, the full-size $\operatorname{purF}_{E}$ gene encoding the protein with both amino acid replacements was obtained. As found in the case of the prs $_{E}$ gene, the SD site optimized for bacilli was introduced into primer F1. The modified $\operatorname{purF}_{E}$ gene was cloned into the pLE1 plasmid under the control of the $\mathrm{P}_{r p s F}$ promoter at the ClaI and EspI sites. The modified $\operatorname{purF}_{E}$ gene was cloned into the pLE2 plasmid containing the prs $_{E}$ gene using the same procedure. The corresponding plasmids were named pLE3 and pLE4 (Table 1). At the final stage, plasmids pLE3 and pLE4 were integrated into the amyE B. subtilis chromosome locus according to the scheme described above (Fig. 4). The strains obtained by integrating plasmids pLE3 and pLE4 were named AM813 and AM815, respectively (Table 1).

\section{Determination of the ability of strains to accumulate AICAR}

The fermentation experiments were carried out under the conditions described in the experimental section, in order to estimate the ability of the strains obtained in this study to accumulate AICAR. The results of these experiments are summarized in Fig. 6.

As follows from the data given in Fig. 6, the initial AM732 strain accumulates almost no AICAR. Only after the purH gene has been removed from the genome of this strain (AM793 strain) is a negligible $(<1 \mathrm{~g} / \mathrm{L})$ accumulation of AICAR in CL observed. The mutations of purR::neo and $\Delta T$-purE (AM788 strain) ensuring complete derepression of the enzymes of purine biosynthesis result in a considerable accumulation of AICAR; up to $4-5 \mathrm{~g} / \mathrm{L}$. A subsequent, almost two-fold enhancement of productivity was observed for the AM811 and AM813 strains expressing one of the mutant desensibilized E. coli proteins - PRPP synthase (prs gene) or 
cgcgaa R1 (EcoRI)

tatgaggatcttcttgcgggcggcggtatggcaggagctaaagaggcaggaaaagtccgc

atactcctagaagaacgccegccgccataccgtcctcgatttctccgtccttttcaggcg

cttgaagggaaagaatatgtggtccaagacggagatgttattcatttccgatttaatgta gaacttcccttcttatacaccaggttctgcctctacaataagtaaaggctaaattacat

$-35 \quad-10 \quad+1$

taggatgcagTTGTAAagggacaagagctttggTATAATataaaattgtgagtaatagaa

atcctacgtcaacatttccctgttctcgaaaccatattatattttaacactcattatctt
Fig. 5. Nucleotide sequence of the rpsF gene promoter. -10 and -35 regions shown in bold uppercase. Positions +1 defined as transcription start of pur-operon. The UP-element of the promoter is boxed. The nucleotide sequence of the primers used for cloning of the promoter are marked in blue. ttattgctccttgcccattatgg

aataacgaggaacgggtaatacc

R2 (BamHI) taggcgc
glutamine-PRPP aminotransferase ( $p u r F$ gene). The maximum accumulation of AICAR (up to $11-13 \mathrm{~g} / \mathrm{L}$ ) was detected for the AM815 strain, which is characterized by the complete derepression of enzymes of purine biosynthesis and simultaneously contains the modified prs $s_{E}$ and $p u r F_{E}$ genes controlled by the $\mathrm{P}_{r p s F}$ promoter.

It is of interest that almost all of the AICAR synthesized in cells is excreted in CL: it was demonstrated in special experiments that the intracellular concentration of AICAR is no higher than $2 \%$ of its concentration within the medium. The mechanism of AICAR excretion remains unknown, although data was obtained indicating that the membrane protein encoded by the pbuE gene is involved in its export from bacilli cells [41].

The different stages in constructing the AICAR producer are directed towards the stimulation of purine nucleotide biosynthesis; therefore, the AICAR that is detected in CL is formed upon dephosphorylation of the AICAR-P nucleotide synthesized de novo, as opposed to being the secondary product of histidine biosynthesis. Since AICAR-P is the natural analogue of AMP, while AICAR is the analogue of adenosine, the increased content of these compounds in producer cells is apparently accompanied by multiple metabolic events (see above); their impact as regards AICAR production is far from clear. In a microorganism, AICAR-P cells serve not only as an intermediate of purine metabolism, but also as a regulatory molecule of all-cell significance. The transformation of AICAR-P into IMP requires the participation of $\mathrm{N} 10$-formyltetrahydrofolate; therefore, the increase in the level of AICAR-P in cells may be a warning of monocarbon metabolism disorder, which earlier has made it possible to regard this nucleotide as an alarmone [42]. Regardless of the fact that prokaryotes do not have a target for AICAR$\mathrm{P}$ that would be similar to animal AMPK, the direction- ality of the physiological action of this AMP analogue is retained in them. In particular, inactivation of the purH gene in Salmonella enterica and AICAR-P accumulation in cells result in the suppression of the activity of fructose-1,6-bisphosphate phosphatase. As a result, the cells lose their ability for gluconeogenesis and stop growing on glycerol and other gluconeogenic substrates [43]. In cells of prokaryotes and lower eukaryotes (e.g., yeast), a certain amount of AICAR-P is formed as a side product of histidine biosynthesis, which allows additional opportunities for the regulation of purine nucleotide biosynthesis [44]. The numerous regulatory bonds of AICAR-P that remain incompletely studied complicate the construction of producing strains and require further investigation.

\section{CONCLUSIONS}

The strain-producer of AICAR, a new drug with potentially wide therapeutic applications, was obtained in studies based on B. subtilis bacteria. The strategy

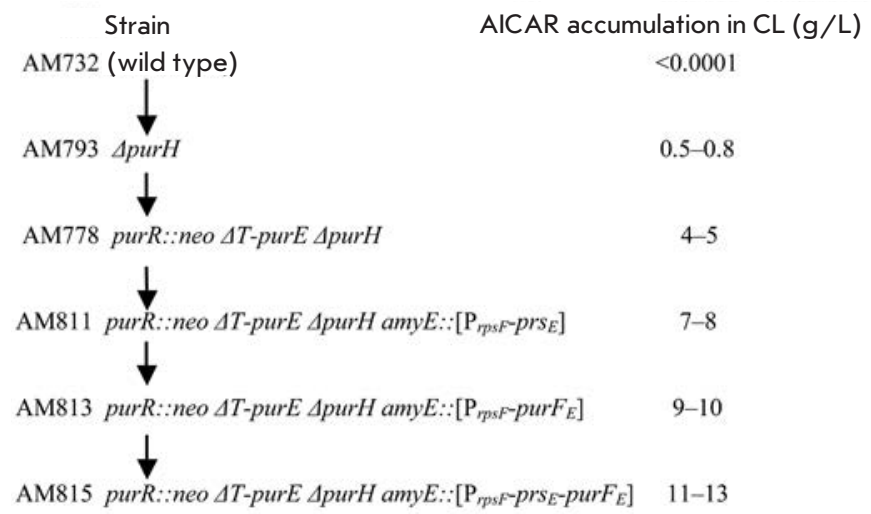

Fig 6. Construction stages of AICAR-producing strains and their productivity. 
obtaining the AICAR producing strain is based on the directed reconstruction of purine metabolism in B. subtilis cells. At the first stage of the study, an insertion was introduced into the purR gene encoding the puroperon repressor protein, and the transcription attenuator was removed from the leader region of the pur-operon, ensuring maximum derepression of the enzymes of de novo purine biosynthesis. The purH gene encoding formyltransferase/IMP-cyclohydrolase was then removed from the bacterial genome. Inactivation of this enzyme disturbs the reaction of AICAR conversion into IMP and results in its accumulation in the cell. At the next stage, the site-directed mutagenesis of prs and purF E. coli genes encoding the key enzymes of the synthesis of purine precursors was carried out in order to obtain mutant variants of these genes that would not be susceptible to retroinhibition by purine nucleotides. Finally, at the last stage, the modified prs and purF E. coli genes were integrated into the $B$. subtilis chromosome under the control of a strong promoter ensuring a high level of expression of these genes in B. subtilis cells. As a result, we obtained a producing strain accumulating $11-13 \mathrm{~g} / \mathrm{L}$ of AICAR in CL.
To summarize, we would like to note that it was reported recently that AICAR had successfully passed the stage IIa of clinical trials as a antitumor agent [45]. AICAR has a positive effect upon chronic lymphocytic leukemia, multiple myeloma, and mantle cell lymphoma. It should be emphasized that the cost of commercial AICAR substances in catalogues varies from US\$100 to 1,000 per gram, which is likely due to the fact that they are prepared by chemical synthesizing. The high cost makes AICAR inaccessible for research and a fortiori for treatment of the metabolic syndrome. The AICAR-producing strain constructed by us may lay the foundations for industrial microbiological production of an affordable AICAR substance that would cost substantially less.

The authors are grateful to N.P. Zakataeva for providing the pNZT1 plasmid and to V.A. Muratova and G.I. Sergeeva for qualified technical assistance.

The work was partially supported by the Dynasty

Foundation for Non-commercial Programs.
REFERENCES

1. Buchanan J.M., Hartman S.C. // Adv. Enzymol. Relat. Areas Mol. Biol. 1959. V. 21. P. 199-261.

2. Zalkin H., Nygaard P. // Escherichia coli and Salmonella. Washington, DC.: ASMI Press, 1996. P. 561-579.

3. Switzer R.L., Zalkin H., Saxild H.H. // Bacillus subtilis and its closest relatives: from genes to cells. Washington, DC.: ASM Press, 2002. P. 255-269.

4. Hardie D.G., Carling D., Carlson M. //Ann. Rev. Biochem. 1998. V. 67. P. 821-855.

5. Hardie D.G., Salt I.P., Hawley S.A., Davies S.P. // Biochem. J. 1999. V. 338. P. 717-722.

6. Rutter G.A., Xavier G.S., Leclerc I. // Biochem. J. 2003.

V. 375. P. 1-16.

7. Gaidhu M.P., Fediuc S., Anthony N.M., So M., Mirpourian

M., Ceddia R.B. // J. Lipid Res. 2009. V. 50. P. 704-715.

8. Swinnen J.V., Beckers A., Brusselmans K., Organe S., Segers J., Timmermans L., Vanderhoydonc F., Deboel L., Derua R., Waelkens E., et al. // Cancer Res. 2005. V. 65. P. 2441-2448.

9. Pold R., Jensen L.S., Jessen N., Buhl E.S., Schmitz O., Flyvbjerg A., Fujii N., Goodyear L.J., Gotfredsen C.F., Brand C.L., et al. // Diabetes. 2005. V. 54. P. 928-934.

10. Campas C., Lopez J.M., Santidrian A.F., Barragan M., Bellosillo B., Colomer D., Gil J. // Blood. 2003. V. 101. P. 36743680 .

11. Sengupta T.K., Leclerc G.M., Hsieh-Kinzer T.T. // Mol. Cancer. 2007. V. 6(46). P. 1-12.

12. Matsuno K., Mori Y., Asahara T. // US Patent 7326546 (2008).

13. Asahara T., Mori Y., Zakataeva N.P., Livshits V.A., Yoshida K., Matsuno K. // Appl. Microb. Biotechnol. 2010. V. 87. P. 2195-2207.
14. Ebbole D.J., Zalkin H. //J. Biol. Chem. 1987. V. 262. P. 8274-8287.

15. Weng M., Nagy P.L., Zalkin H. // Proc. Natl. Acad. Sci. USA. 1995. V. 92. P. 7455-7459.

16. Ebbole D.J., Zalkin H. // J. Biol. Chem. 1988. V. 263.

P. 10894-10902.

17. Johansen L.E., Nygaard P., Lassen C., Agerso Y., Saxild H.H. // J. Bacteriol. 2003. V. 185. P. 5200-5209.

18. Mironov A.S., Gusarov I., Rafikov R., Lopez L.E., Shatalin K., Kreneva R.A., Perumov D.A., Nudler E. // Cell. 2002.

V. 111. P. 747-756.

19. Nudler E., Mironov A. S. // Trends Biochem. Sci. 2004.

V. 29. P. 11-17.

20. Mandal M., Boese B., Barrick J.E., Winkler W.C., Breaker R.R. // Cell. 2003. V. 113. P. 577-586.

21. Lobanov K.V., Korol'kova N.V., Eremina, S. Yu., Errais Lopes L., Mironov A.S. // Rus. J. Genetics. 2011. V. 47. P. 785-793.

22. Hove-Jensen B., Nygaard P. // Eur. J. Biochem. 1982. V. 126. P. 327-332.

23. Meyer E., Switzer R.L. // J. Biol. Chem. 1979. V. 254. P. 5397-5402.

24. Turnbough C.L., Switzer R.L. // J. Bacteriol. 1975. V. 121. P. 108-114.

25. Zhou G., Smith J.L., Zalkin H. // J. Biol. Chem. 1994.

V. 269. P. 6784-6789.16. C.L.

26. Anagnostopoulos C., Spizizen J. // J. Bacteriol. 1961. V. 81. P. 741-746.

27. Yoshikawa H., Sueoka N. // Proc. Nat. Acad. Sci. USA. 1963. V. 49. P. 559-566.

28. Christiansen L.C., Schou S., Nygaard P., Saxild H.H. // J.

Bacteriol. 1997. V. 179. P. 2540-2550.

29. Blattner F.R., Plunkett G. 3rd, Bloch C.A., Perna N.T., 


\section{RESEARCH ARTICLES}

Burland V., Riley M., Collado-Vides J., Glasner J.D., Rode C.K., Mayhew G.F., et al. // Science. 1997. V. 277. P. 14531474.

30. Zakataeva N.P., Nikitina O.V., Gronskiy S.V., Romanenkov D.V., Livshits V.A. //Appl. Microbiol. Biotechnol. 2010. V. 85. P. 1201-1209.

31. Sambrook J., Fritsch E.F., Maniatis T. Molecular cloning: a laboratory manual, 2nd ed. N.Y. Cold Spring Harbor: Cold Spring Harbor Lab. Press. 1989.

32. Saito H., Miura K.I. // Biochim. Biophys. Acta. 1963. V. 42. P. 619-629.

33. Sanger F., Nicklen S., Coulson A.R. // Proc. Natl. Acad. Sci. USA. 1977. V. 74. P. 5463-5467.

34. Shatalin K.Y., Neyfakh A.A. // FEMS Microbiol. Lett. 2005. V. 245. P. 315-319.

35. Arnvig K., Hove-Jensen B., Switzer R.L. // Eur. J. Biochem. 1990. V. 192. P. 195-200.

36. Hove-Jensen B., Harlow K.W., King C.J., Switzer R.L. // J. Biol. Chem. 1986. V. 261. P.6765-6771.

37. Henkin T.M. // Bacillus subtilis and other gram-positive bacteria: biochemistry, physiology, and molecular genetics
/ Eds. Sonenshein A.L., Hoch J.A., Losick R. Washington, D.C.: American Soci. Microbiol., 1993. P. 669-682.

38. Linder C., Nijland R., Hartskamp M. // J. Bacteriol. 2004. V. 186. P. 1097-1105.

39. Estrem S.T., Gaal T., Ross W. Gourse R.L. // Proc. Natl. Acad. Sci. USA. 1998. V. 95. P. 9761-9766.

40. Chen S., Tomchick D.R., Wolle D., Hu P., Smith J.L., Switzer R.L., Zalkin H. // Biochemistry. 1997. V. 36. P. 1071810726.

41. Sheremet A.S., Gronskiy S.V., Akhmadyshin R.A., Novikova A.E., Livshits V.A., Shakulov R.S., Zakataeva N.P. // J. Ind. Microbiol. Biotechnol. 2011. V. 38. P. 65-70.

42. Bochner B.R., Ames B.N. // Cell. 1982. V. 29. P. 929-937.

43. Dougherty M.J., Boyd J.M., Downs D.M. // J. Biol. Chem. 2006. V. 28. P. 33892-33899.

44. Rebora K., Laloo B., Daignan-Fornier B. // Genetics. 2005. V. 170. P. $61-70$

45. The Advancell Company Press Release on 16.02.2011. 